\title{
Quantum melting of two-component Rydberg crystals
}

\author{
Zhihao Lan, Weibin Li, and Igor Lesanovsky \\ School of Physics and Astronomy, University of Nottingham, Nottingham NG7 2RD, United Kingdom \\ and Centre for the Mathematics and Theoretical Physics of Quantum Non-equilibrium Systems, University of Nottingham, \\ Nottingham NG7 2RD, United Kingdom
}

(Received 16 May 2016; published 11 November 2016)

\begin{abstract}
We investigate the quantum melting of one-dimensional crystals that are realized in an atomic lattice in which ground state atoms are laser excited to two Rydberg states. We focus on a regime where both, intra- and interstate density-density interactions as well as coherent exchange interactions contribute. We determine stable crystalline phases in the classical limit and explore their melting under quantum fluctuations introduced by the excitation laser as well as two-body exchange. We find that within a specific parameter range quantum fluctuations introduced by the laser can give rise to a devil's staircase structure which one might associate with transitions in the classical limit. The melting through exchange interactions is shown to also proceed in a steplike fashion, in the case of small crystals, due to the proliferation of Rydberg spin waves.
\end{abstract}

DOI: 10.1103/PhysRevA.94.051603

Introduction. A long-standing topic in the study of condensed matter physics is the melting of low-dimensional crystals that consist of interacting particles. In two dimensions (2D), it is widely accepted that thermally driven melting from a crystal to a liquid is a two-step procedure mediated by a hexatic phase according to the Kosterlitz, Thouless, Halperin, Nelson, and Young (KTHNY) scenario [1]. Interestingly, melting of quasi-one-dimensional (quasi-1D) crystals can proceed through either first- or second-order transitions, depending on the system parameters [2]. Both situations are different from three-dimensional crystals which melt via a first-order transition as predicted by Landau's mean-field theory [3]. Despite this broad understanding in the classical limit only little is known about the melting of crystals through quantum fluctuations.

In recent years there has been a growing effort to address the dimension-dependent crystallization and its melting by using ultracold atomic and molecular gases. In 2D systems of cold polar molecules first-order superfluid-to-crystal transitions $[4,5]$ and the effect of quantum fluctuations on the formation of a hexatic phase [6,7] have been theoretically investigated. In systems of Rydberg atoms, crystalline phases [8-17] and their melting [18-20] have attracted intensive attention and the experimental preparation of crystalline ground states (GSs) was reported [21] recently. The mechanism behind the quantum melting of a single-component Rydberg crystal in 1D is a two-stage process [18] (similar to the KTHNY scenario), where a commensurate solid with true long-range order melts to a floating solid with quasi-long-range order, and finally to a liquid phase.

The goal of this work is to shed light on melting mechanisms of $1 \mathrm{D}$ crystals in a physical setting in which two species of Rydberg atoms are excited. Such multicomponent Rydberg gases currently receive much attention [22-30]. More importantly, the choice of this setting is that it permits the investigation of local and nonlocal quantum melting, driven by single- and two-body processes, respectively. Atoms in Rydberg states experience strong van der Waals (vdW) type spin flip-flop (exchange) interactions, which can be comparable to their inter- and intrastate density-density vdW interactions [31-33]. Crystalline phases that are stabilized by the density-density interaction are melted by the laser coupling (local melting) and spin exchange (nonlocal melting), respectively. In the case of the local melting, the order parameter undergoes either a smooth or an abrupt (first-order) transition. In the latter situation, the steplike structure resembles a devil's staircase that is typically observed in classical crystals [34] but not in the quantum regime. To shed light on the nonlocal melting process, we consider a parameter regime where only Rydberg states contribute to the many-body GS. Here the 1D Rydberg gas is described by the Heisenberg $X X Z$ model. We demonstrate that a small Rydberg crystal is melted by the proliferation of delocalized Rydberg spin waves, which also gives rise to discontinuous changes of the order parameter. Eventually, we identify specific configurations with which the quantum melting explored in this work can be realized experimentally with rubidium atoms.

The system. We consider atoms held in a 1D deep optical lattice (lattice spacing $d$ and number of lattice sites $L$ ) with one atom per site. Each atom consists of three electronic states $|0\rangle,|1\rangle$, and $|2\rangle$. As shown in Fig. 1(a), the atomic GS $|0\rangle$ is laser coupled to the Rydberg state $|1\rangle(|2\rangle)$ with Rabi frequency $\Omega_{1}\left(\Omega_{2}\right)$ and detuning $\Delta_{1}\left(\Delta_{2}\right)$. The detuning $\Delta_{1}$ $\left(\Delta_{2}\right)$ effectively acts as a chemical potential for the state $|1\rangle(|2\rangle)$. For two Rydberg atoms located on sites $j$ and $k$, we parametrize their intrastate and interstate density-density interaction by $V_{j, k}^{(\alpha)}=V_{\alpha} /(j-k)^{6}$ and $U_{j, k}=U /(j-k)^{6}$, and the exchange interaction by $W_{j, k}=W /(j-k)^{6}$, where $V_{\alpha}=C_{\alpha} / d^{6}(\alpha=1,2), U=C_{\mathrm{d}} / d^{6}$ and $W=C_{\mathrm{e}} / d^{6}$ denote the corresponding nearest-neighbor (NN) interactions. Here $C_{\alpha}, C_{\mathrm{d}}$, and $C_{\mathrm{e}}$ are the respective dispersion coefficients. This yields the following Hamiltonian for the system, which we write as the sum of a classical $\left(H_{\mathrm{c}}\right)$ and a quantum $\left(H_{\mathrm{q}}\right)$ term:

$$
\begin{aligned}
H & =H_{\mathrm{c}}+H_{\mathrm{q}}, \\
H_{\mathrm{c}} & =\sum_{l>k, \alpha}\left[V_{\alpha} \frac{n_{k}^{(\alpha)} n_{l}^{(\alpha)}}{(l-k)^{6}}+\frac{U}{2} \sum_{\alpha^{\prime} \neq \alpha} \frac{n_{k}^{(\alpha)} n_{l}^{\left(\alpha^{\prime}\right)}}{(l-k)^{6}}\right]-\sum_{k, \alpha} \Delta_{\alpha} n_{k}^{(\alpha)}, \\
H_{\mathrm{q}} & =\sum_{k}\left[W \sum_{l>k} \frac{\sigma_{k}^{(+)} \sigma_{l}^{(-)}+\sigma_{k}^{(-)} \sigma_{l}^{(+)}}{(l-k)^{6}}+\Omega_{1} \sigma_{k}^{(1)}+\Omega_{2} \sigma_{k}^{(2)}\right] .
\end{aligned}
$$



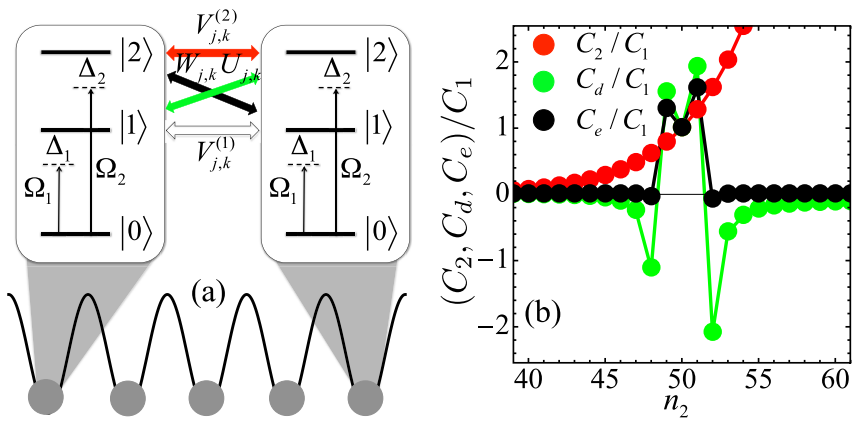

FIG. 1. (a) The system. Atoms are held in a 1D optical lattice. The atomic ground state $|0\rangle$ is laser excited to the Rydberg state $|1\rangle(|2\rangle)$ with Rabi frequency $\Omega_{1}\left(\Omega_{2}\right)$ and detuning $\Delta_{1}\left(\Delta_{2}\right)$. For two Rydberg atoms sitting at site $j$ and $k$, their intrastate, interstate, and exchange interactions are $V_{j, k}^{(\alpha)}(\alpha=1,2), U_{j, k}$, and $W_{j, k}$, respectively. See text for details of the interaction potential. (b) Dispersion coefficients of the intrastate $\left(C_{1}, C_{2}\right)$ and interstate $\left(C_{\mathrm{d}}\right) \mathrm{vdW}$ interaction as well as exchange interaction $\left(C_{\mathrm{e}}\right)$ for two Rydberg $S$ states of rubidium as a function of the principal quantum number $n_{2}$. We fix $n_{1}=50$, which yields $C_{1}=13.7 \mathrm{GHz} \mu \mathrm{m}^{6}$. Note the interstate density-density vdW interaction $U_{j, k}$ and exchange interaction $W_{j, k}$ appear in the classical and quantum parts of the Hamiltonian, respectively [see Eq. (1)].

The local operators on site $j$ are given by $n_{j}^{(\alpha)}=$ $|\alpha\rangle_{j}\left\langle\alpha\left|, \quad \sigma_{j}^{(\alpha)}=\right| \alpha\right\rangle_{j}\langle 0|+| 0\rangle_{j}\left\langle\alpha\left|, \quad \sigma_{j}^{(+)}=\right| 2\right\rangle_{j}\langle 1|, \quad \sigma_{j}^{(-)}=$ $|1\rangle_{j}\langle 2|$, where $\alpha=1,2$ denotes the two Rydberg states. We denote $H_{\mathrm{c}}$ as classical as it contains only diagonal operators $n_{j}^{(\alpha)}$ acting on the local single particle Hilbert spaces. The quantum part $H_{\mathrm{q}}$, on the other hand, contains the off-diagonal operators $\sigma_{j}^{(\alpha)}, \sigma_{j}^{(+)}$, and $\sigma_{j}^{(-)}$. There is a large flexibility in tuning laser parameters $\left(\Delta_{1}, \Delta_{2}, \Omega_{1}, \Omega_{2}\right)$. The strength of the vdW interaction is fixed by the specific choice of Rydberg states (see discussion towards the end of the Rapid Communication). For convenience, energies will be scaled with respect to the $\mathrm{NN}$ interaction $V_{1}$ in the following.

Classical two-component Rydberg crystals. In the following we will investigate the nature of the GS in the classical limit, $H_{\mathrm{q}}=0$. Note that certain aspects of this have been addressed by some of us in previous works [35,36], which were, however, limited to very specific parameter values, i.e., $V_{2} \gg V_{1}$ and $U=W=0$. There it was shown that the presence of the strongly interacting species $\left(V_{2}\right)$ can lead to frustration effects preventing the weakly interacting species $\left(V_{1}\right)$ from assuming its lowest energy configuration.

To understand the coarse structure of the classical crystalline GS configurations, we will for the moment approximate the vdW interactions as $\mathrm{NN}$ interactions. Using the technique of irreducible blocks (see [37] for an introduction to the technique and [38] for the original reference), seven possible irreducible blocks $\{0,1,2,01,02,12,012\})$ are identified, which provide the unit-cell structure of GS crystals. Their energy densities can be found analytically and are summarized in Table I. Note that the phase VII cannot be the GS of the system for any set of parameters, due to $E_{\mathrm{II}}+E_{\mathrm{IV}}+E_{\mathrm{VI}}=$ $3 E_{\mathrm{VII}}$, i.e., its energy is always larger than at least one of the other phases.
TABLE I. The seven possible crystalline phases of $H_{\mathrm{c}}$ and their corresponding energy densities.

\begin{tabular}{lcl}
\hline \hline Label & Configuration & \multicolumn{1}{c}{ Energy density } \\
\hline I & $000 \cdots$ & $E_{\mathrm{I}}=0$ \\
II & $101010 \cdots$ & $E_{\mathrm{II}}=-\Delta_{1} / 2$ \\
III & $111 \cdots$ & $E_{\mathrm{III}}=-\Delta_{1}+V_{1}$ \\
IV & $202020 \cdots$ & $E_{\mathrm{IV}}=-\Delta_{2} / 2$ \\
V & $222 \cdots$ & $E_{\mathrm{V}}=-\Delta_{2}+V_{2}$ \\
VI & $121212 \cdots$ & $E_{\mathrm{VI}}=\left(-\Delta_{1}-\Delta_{2}+2 U\right) / 2$ \\
VII & $012012012 \cdots$ & $E_{\mathrm{VII}}=\left(-\Delta_{1}-\Delta_{2}+U\right) / 3$ \\
\hline \hline
\end{tabular}

In Figs. 2(a)-2(e), we present phase diagrams in the $\Delta_{1}-\Delta_{2}$ plane for different values of the interstate interaction $U$. In each situation, the crystal configuration can be changed from one containing no Rydberg excitation, to a single-component or a two-component Rydberg crystal, by modifying the laser detuning $\Delta_{1}$ or $\Delta_{2}$. When comparing these panels, the relative areas occupied by different phases are modified by $U$. For examples, the region occupied by the composite crystalline phase VI first shrinks and finally disappears when $U$ increases from $-5 V_{1}$ to $3 V_{1}$ (the phase diagram no longer changes when $\left.U \geqslant 3 V_{1}\right)$.

Let us now investigate the effect of the tail of the vdW interaction on the classical GS phase diagrams of Figs. 2(a)2(e). In 1D (single-component) Ising models, it has been shown that such algebraically decaying potentials lead to the formation of a devil's staircase [34]. This is a fractal structure whose steps (or plateaus) are defined as the stability regions of configurations with specific rational filling fractions (density of excitations). Such structure is also formed in the two-component system in the vicinity of the phase boundaries

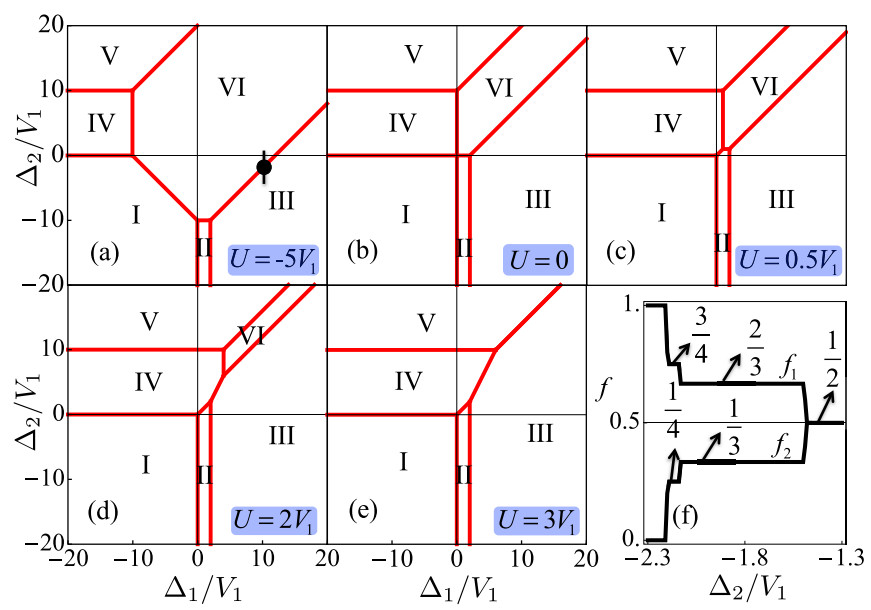

FIG. 2. Ground state phase diagrams of the classical Hamiltonian $H_{\mathrm{c}}$ for $U=-5 V_{1}(\mathrm{a}), 0(\mathrm{~b}), 0.5 V_{1}$ (c), $2 V_{1}(\mathrm{~d})$, and $3 V_{1}$ (e), obtained by explicitly checking which configuration of the crystalline phasesas listed in Table I-has the lowest energy density for given $\Delta_{1,2}$. We consider here only the $\mathrm{NN}$ interaction and set $V_{2}=5 V_{1}$. Panel (f) shows a magnification of the staircase structure in the vicinity of $\left(\Delta_{1}, \Delta_{2}\right)=(10,-2) V_{1}$ along $\Delta_{2}$ as marked in (a) that emerges when including the vdW tail. Here we display the populations, $f_{\alpha}=$ $\sum_{j=1}^{L} n_{j}^{(\alpha)} / L$, of the atomic state $|\alpha\rangle$. 
displayed in Figs. 2(a)-2(e). As an example, we calculate stable classical crystalline phases in the transition region between the phases III and VI, around the point marked in Fig. 2(a). The calculation is done by explicitly checking which rational filling fraction, of the form $f=p / q$ (with $p \leqslant q$ and maximal $q=13$ ) of an infinite system with period $q$, has the lowest energy per site [17]. Performing calculations with large $q>13$ makes the numerics more tedious and also adds little information to the coarse structure of the staircase as stable configurations with large $q$ normally correspond to high commensurate phases with very narrow steps. In Fig. 2(f) we display the populations of the atomic states, $f_{\alpha}=$ $\sum_{j=1}^{L} n_{j}^{(\alpha)} / L,(\alpha=0,1,2)$ - which in the following serve as an order parameter - as a function of $\Delta_{2}$. We observe a number of steps-reminiscent of a devil's staircase structure—on each of which the components of order parameter assume rational values different from those corresponding to the phases of Table I. Hence each plateau represents a new crystalline phase with narrower stability region. For example, the second largest plateau corresponds to $f_{1}=2 / 3$ (or $f_{2}=1 / 3$ ). Its length along the $\Delta_{2}$ axis is $0.63 V_{1}$, which is only about $2 \%$ of the phase VI. An open question is whether our two-component system can indeed form a complete devil's staircase [34].

Laser induced local melting. It was found that the laser induced melting of a single-component Rydberg crystal is a continuous and two-stage process $[18,19]$. In contrast, we will illustrate here that such local melting of a two-component Rydberg crystal can proceed via a series of discontinuous transitions. To this end, we consider the case in which the exchange interaction between Rydberg states can be neglected. We begin by numerically diagonalizing a finite size system with $L=10$. The parameters of the laser driving the $|0\rangle-|1\rangle$ transition [see Fig. 1(a)] are fixed to $\Omega_{1}=0$ and $\Delta_{1}=10 V_{1}$ such that the accessible classical phases are given by the configurations III, V, and VI [see Figs. 2(a)-2(d)]. The crystal melting is then solely effectuated by the second laser whose Rabi frequency $\Omega_{2}$ we vary. With this particular set of parameters (i.e., $\Omega_{1}=0$ and varying $\Omega_{2}$ ), atoms in state $|1\rangle$ remain essentially "classical" while the states $|2\rangle$ and $|0\rangle$ form a superposition that ultimately leads to the quantum melting of classical crystalline states. We will discuss the effect of finite $\Omega_{1}$ on the melting process further below.

The components $f_{\alpha}$ of the order parameter are shown in Figs. 3(a)-3(c). Additional cuts along $\Delta_{2}=-10 V_{1}$ are provided in Fig. 3(d). The data indicate a number of sharp jumps reminiscent of first-order transitions that start from the classical limit $\left(\Omega_{2}=0\right)$ and extend into the quantum regime. For example, in Fig. 3(d), when $\Omega_{2}$ is smaller than a critical value $\Omega_{\mathrm{L}} \approx 11.9 V_{1}$, the GS is formed by atoms in state $|1\rangle$ - the phase III-and the laser $\Omega_{2}$ in fact has no effect. However, once $\Omega_{2}>\Omega_{\mathrm{L}}$, all three atomic states are populated suddenly, such that $f_{1}=0.5$ remains constant, while the other two vary smoothly with respect to $\Omega_{2}$. By further increasing $\Omega_{2}$ one reaches a second critical value $\Omega_{\mathrm{H}} \approx 15 V_{1}$, from which onwards the population of $|1\rangle$ is completely suppressed and $f_{0}$ and $f_{2}$ change smoothly. Contrary to the above situation, melting of the V phase [Fig. 3(e)] proceeds smoothly since this corresponds to the melting of a single-component Rydberg crystal [18] that only involves the states $|2\rangle$ and $|0\rangle$.

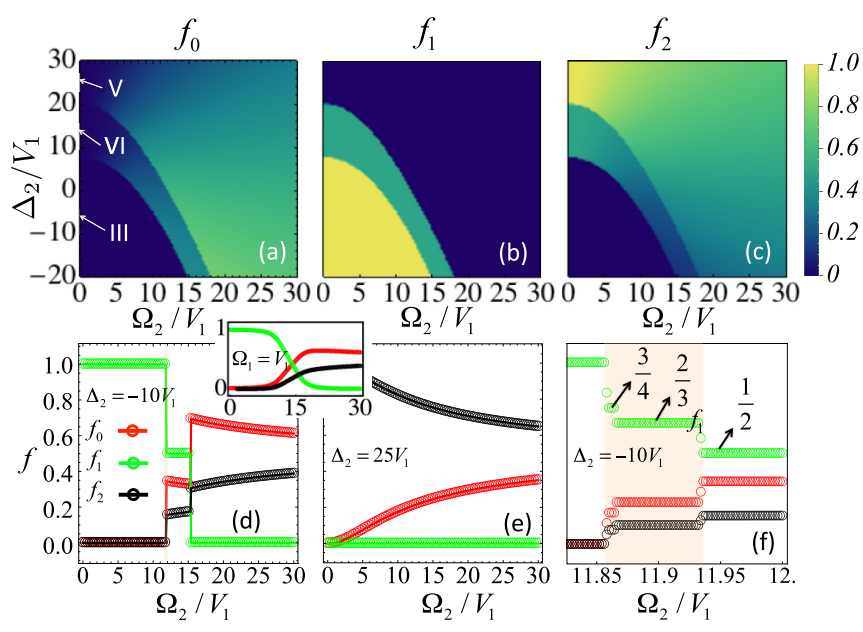

FIG. 3. Laser induced local melting with $\Omega_{1}=0$ while varying $\Omega_{2}$. (a)-(c) Populations $f_{0}, f_{1}$, and $f_{2}$. The sharp change of populations clearly demonstrates the first-order nature of the laser induced melting (in the classical limit the phases III, V, and VI are marked by arrows). The considered Rydberg states are $n_{1}=50$ and $n_{2}=57$ (such that $W \simeq 0$; see Fig. 1) and $\Delta_{1}=10 V_{1}$. The data is obtained by the exact diagonalization of a finite lattice with $L=10$ under periodic boundary conditions, where the tail of the vdW interaction is included in the numerics. In panel (d), the first-order transition is clearly visible. In panel (e), a smooth melting transition is shown. The mean-field results (solid lines) with only NN interactions agree well with the numerical diagonalization results (circles). (f) Magnification of the devil's staircase in the vicinity of $\Omega_{2}=11.9 V_{1}$ as shown in panel (d). To resolve next largest plateaus at $f_{1}=2 / 3$ and $3 / 4$, a larger lattice with $L=12$ was chosen for the calculation. Note that the staircase structure vanishes for finite values of $\Omega_{1}$ as can be seen in the inset of panel (d). Here $\Omega_{1}=V_{1}$ with all other parameters being the same as in (d).

The observed phase diagram is largely captured by a mean-field (MF) theory where we write the site-decoupled GS wave function as $|\Psi\rangle=\prod_{i} \otimes\left(a_{i}|0\rangle_{i}+b_{i}|1\rangle_{i}+c_{i}|2\rangle_{i}\right)$ [39]. To illustrate the main mechanism we will again consider for the moment only $\mathrm{NN}$ interactions and as the unit cell occupies at most two sites with only NN interactions (see Table I), the period of the wave function is two sites. The order parameter obtained from the MF calculation is in very good agreement with the diagonalization results [see Figs. 3(d) and 3(e)]. MF further corroborates the first-order nature of the observed transitions: when $0<\Omega_{2}<\Omega_{\mathrm{L}}$, the wave function of a unit cell is given by a simple Fock state $\left|\psi_{A}\right\rangle=|11\rangle$. However, the wave function becomes $\left|\psi_{B}\right\rangle=\alpha|10\rangle+\beta|12\rangle$ ( $\alpha$ and $\beta$ are normalization constants) when $\Omega_{\mathrm{L}}<\Omega_{2}<\Omega_{\mathrm{H}}$. The order parameter jumps at $\Omega_{\mathrm{L}}$ as the two wave functions cannot be smoothly connected by merely varying $\alpha$ and $\beta$. This also highlights the nature of the first-order transitions driven by $\Omega_{2}$. the $\Omega_{2}$ term of the Hamiltonian is minimized by a superposition of states $|0\rangle$ and $|2\rangle$. Increasing $\Omega_{2}$ (across $\Omega_{\mathrm{L}}$ ) makes phase III energetically unfavorable and leads to a partially crystalline phase. Here one of every two sites is occupied by atoms in state $|1\rangle$ and the other one is in a superposition of states $|0\rangle$ and $|2\rangle$. This is clearly different from the first-order transitions observed in the classical limit where no superposition 
happens. Consequently, this partially crystalline phase features both crystalline antiferromagnetic correlations for state $|1\rangle$ and exponentially decaying density-density correlations for state $|2\rangle$.

Though driving by a quantum term $\Omega_{2}$, the tail of the $\mathrm{vdW}$ interaction leads to the emergence of a devil's staircase in the vicinity of the transition points for state $|1\rangle$, which behaves classically as $\Omega_{1}=0$. The corresponding numerical data around $\Omega_{2}=\Omega_{\mathrm{L}}$ is shown in Fig. 3(f). Here multiple plateaus emerge between the two main plateaus corresponding to $f_{1}=1$ and $f_{1}=0.5$. Transitions between plateaus proceed similarly to the discussion above: on each plateau atoms in the state $|1\rangle$ form a crystalline structure, whose staircase has the same pattern as its classical counterpart [see Fig. 2(f)]. However, the sites that were originally occupied by an atom in state $|2\rangle$ now enter a superposition state and "melt." We would like to point out that the staircase of $f_{1}$ displayed in Fig. 3(f), exhibits the same plateaus as its classical counterpart given in Fig. 2(f). The steps in the population are thus physical and a consequence of the "classical species" (in state |1 $\rangle$ ) adapting its density in order to achieve the overall lowest energy state of the system. Quantum fluctuations introduced by a finite coupling $\Omega_{1}$ smear out the staircase. This is shown in the inset of Fig. 3(d).

Exchange interaction induced nonlocal melting. To discuss the nonlocal melting we consider a regime where only the two Rydberg states play roles in the physics. This is achieved when $\Omega_{\alpha}=0$ and $\Delta_{\alpha}(\alpha=1,2)$ is sufficiently large, such that classically the GS can only be one of the phases III, V, and VI. With this choice of parameters, the state $|0\rangle$ is never populated even when the many-body GS is away from the classical limit.

First we focus on a simplified situation in which the three relevant interactions are of equal strength, i.e., $V_{2}=U=V_{1}$. By numerically diagonalizing the Hamiltonian (1), we obtain the GS phase diagram of a small crystal of $L=10$. According to the population $f_{1}$ plotted in Fig. 4(a), the system is in the crystalline phase III (V) when $\Delta_{2}$ is negative (positive) and $\left|\Delta_{2}\right| \gg W$. From the crystalline phase, $f_{1}$ jumps abruptly when we scan either $W$ or $\Delta_{2}$. For example, when increasing $\Delta_{2}$ along the vertical arrow shown in Fig. 4(a), the phase III melts at the first jump and the new many-body GS contains one more excitation in state $|2\rangle$. This process repeats at every jump until $f_{2}=1\left(f_{1}=0\right)$, i.e., the phase $\mathrm{V}$.

To understand this melting pattern, we project Hamiltonian (1) to the subspace of the two Rydberg states and consider only NN interactions for simplicity. This reduces the system to a spin-1/2 Heisenberg $X X$ model with a field along the $\sigma_{z}$ direction,

$$
H_{X X}=\sum_{i} \frac{W}{2}\left(\sigma_{i}^{x} \sigma_{i+1}^{x}+\sigma_{i}^{y} \sigma_{i+1}^{y}\right)+h \sigma_{i}^{z}+C,
$$

where $\quad h=\left(-V_{1}+V_{2}+\Delta_{1}-\Delta_{2}\right) / 2, \quad C=\left(V_{1}+V_{2}+\right.$ $\left.2 U-2 \Delta_{1}-2 \Delta_{2}\right) L / 4$, and $\sigma_{i}^{\xi}(\xi=x, y, z)$ are the Pauli matrices for the two Rydberg states on site $i$.

This Hamiltonian can be analytically solved which permits one to show that the melting of phase III (V) is due to a proliferation of Rydberg spin wave states. To be concrete, we will focus in the following on the melting of phase III, whose wave function is given by $\left|\Psi_{\mathrm{G}}\right\rangle=\Pi_{k}^{L} \otimes|1\rangle_{k}$. The eigenstates $\left|\Psi_{N}\right\rangle$

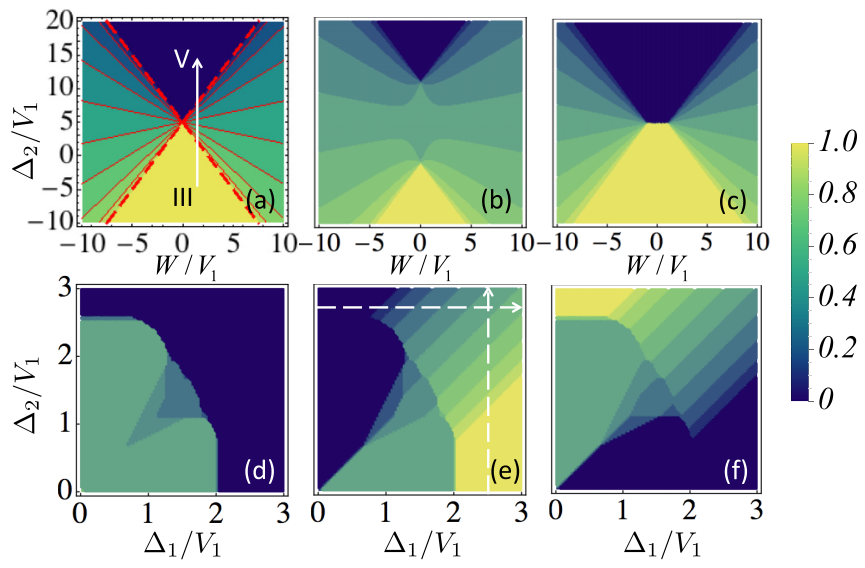

FIG. 4. Population $f_{1}$ for $U=V_{1}$ (a), $-2 V_{1}$ (b), and $2 V_{1}$ (c). The red (solid and dashed) lines in (a) are the analytic results with only $\mathrm{NN}$ interactions from Eq. (3). Other parameters are $V_{2}=V_{1}$, $\Omega_{1}=\Omega_{2}=0$, and $\Delta_{1}=5 V_{1}$. (d)-(f) Populations $f_{0}, f_{1}$, and $f_{2}$ for the experimental relevant case: two Rydberg $S$ states of rubidium with principal quantum numbers $n_{1}=50$ and $n_{2}=51$ [see data in Fig. 1(b)]. The melting of phase III (V) can be probed by changing $\Delta_{2}\left(\Delta_{1}\right)$ along the vertical (horizontal) arrow in (e). The tail of the $\mathrm{vdW}$ interaction is included in the numerical diagonalizations.

of Eq. (2) that contain a fixed number $N$ of spin excitations in state $|2\rangle$ can be explicitly calculated. For example, for $N=1$, $\left|\Psi_{1}\right\rangle=1 / \sqrt{L} \sum_{j} \sigma_{j}^{(+)}\left|\Psi_{\mathrm{G}}\right\rangle$ is a spin wave where the single excitation in state $|2\rangle$ is shared by all the atoms in the lattice. From the eigenenergies $E_{N}^{\min }=V_{1}-\Delta_{1}(L-N) / L-\Delta_{2} N / L-$ $2 W \sin (N \pi / L) /[L \sin (\pi / L)]$ [40], we obtain the transition from $N$ to $N+1$ excitations by varying the detuning $\Delta_{2}$,

$$
\Delta_{2}=\Delta_{1}-\frac{2 W}{\sin (\pi / L)}\left[\sin \frac{(N+1) \pi}{L}-\sin \frac{N \pi}{L}\right] .
$$

These steps [see red solid and dashed lines in Fig. 4(a)] agree well with the position steps that were found in the numerics. The analytical results indicate that the crystal phase $\left|\Psi_{\mathrm{G}}\right\rangle$ (i.e., phase III) switches to the delocalized spin wave state $\left|\Psi_{1}\right\rangle$ when we increase $\Delta_{2}$ (fixing $W$ ). The transition points, determined by $\Delta_{2}=\Delta_{1}-2 W$, are highlighted by the two dashed lines in Fig. 4(a). Note, that the steplike structure appears only for small sizes which are in fact relevant for current experiments [21]. For macroscopic sizes the energy gaps between spin wave states vanish and the excitation density will vary continuously as a function of $W$.

Away from the special point $V_{2}=U=V_{1}$, the system is described by a Heisenberg $X X Z$ model, $H_{X X Z}=H_{X X}+$ $V_{z} \sum_{i} \sigma_{i}^{z} \sigma_{i+1}^{z}$ with $V_{z}=\left(V_{1}+V_{2}-2 U\right) / 4$, whose engineering in controllable quantum systems has attracted increased attention recently [41-46]. Here the presence of the $\sigma_{i}^{z} \sigma_{i+1}^{z}$ interaction terms changes the phase diagram structure. Two examples with $U= \pm 2 V_{1}$ and $V_{2}=V_{1}$ are shown in Figs. 4(b) and $4(c)$. Although the phase boundary changes, the melting of the crystalline phase III (V) also proceeds through the proliferation of spin wave excitations, which has been verified by analyzing both the Hamiltonian (1) and the effective Hamiltonian $H_{X X Z}$.

Experimental implementation of the quantum melting. Local melting is induced by controlling the excitation strength 
of Rydberg states. This has been realized in optical lattices or microtraps by several experimental groups [21,30,47-56]. In the following, we will focus on how to realize the nonlocal melting, which solely depends on the presence of two-body exchange interactions. One possible way to establish strong exchange interactions is to choose two Rydberg $S$ states whose principal quantum numbers $n_{\alpha}$ differ by 1 [33]. For example, dispersion coefficients for rubidium and $n_{1}=50$ and $n_{2}=51$ are $C_{1}=13.7 \mathrm{GHz} \mu \mathrm{m}^{6}, C_{2}=17.4 \mathrm{GHz} \mu \mathrm{m}^{6}$, $C_{d}=26.4 \mathrm{GHz} \mu \mathrm{m}^{6}$, and $C_{e}=21.9 \mathrm{GHz} \mu \mathrm{m}^{6}$. Alternatively, one could utilize the so-called Förster resonance to generate strong exchange interaction. In this case one can even tune the two-body interaction from a van der Waals to dipolar type with external electric fields [57].

In the following, we will illustrate how to observe the nonlocal melting by using an example with the Rydberg $50 \mathrm{~S}$ and $51 S$ states. For lattice spacing $d=3 \mu \mathrm{m}$ [51], we obtain a NN interaction of $V_{1} \approx 18.8 \mathrm{MHz}$. Since the two-body interactions are fixed, the nonlocal melting can be studied by changing the laser detunings $\Delta_{1}$ and $\Delta_{2}$. In Figs. 4(d)-4(f), we present populations $f_{\alpha}$ of the states $|0\rangle,|1\rangle$, and $|2\rangle$ calculated with these parameters. Note that compared to the ideal situation shown in Figs. 4(a)-4(c), the state $|0\rangle$ is in fact populated in certain parameter region [see lower-left corner in Fig. 4(d)]. To probe the melting through spin wave proliferation of the
Rydberg state, we have to avoid this parameter region. For example, one finds that $f_{1}=1$ when $\Delta_{1}=2.5 V_{1}$ and $\Delta_{2}=0$. From here, we can then observe the melting of phase III by increasing $\Delta_{2}$ as indicated by the vertical arrow in Fig. 4(e) [see also Fig. 4(a)].

Outlook. The goal of our study was to shed light on the nature of multicomponent Rydberg crystals and in particular their melting under different kinds of quantum fluctuations. We found that, surprisingly, the quantum melting can proceed via first-order phase transitions through a sequence of steps on a devil's staircase. The second melting mechanism, which proceeds through the proliferation of spin waves, could potentially be employed for the deterministic creation of single- and multiphoton states [58-60].

Acknowledgments. We thank R. M. W. van Bijnen, R. Nath, and T. Pohl for discussions, and M. Marcuzzi for comments on the manuscript. The research leading to these results has received funding from the European Research Council under the European Union's Seventh Framework Programme (FP/2007-2013)/ERC Grant Agreement No. 335266 (ESCQUMA), the EU-FET Grant No. 512862 (HAIRS), the H2020-FETPROACT-2014 Grant No. 640378 (RYSQ), and EPSRC Grant No. EP/M014266/1. W.L. is supported through the Nottingham Research Fellowship by the University of Nottingham.
[1] J. M. Kosterlitz and D. J. Thouless, J. Phys. C 5, L124 (1972); B. I. Halperin and D. R. Nelson, Phys. Rev. Lett. 41, 121 (1978); A. P. Young, Phys. Rev. B 19, 1855 (1979).

[2] Y. Levin and K. A. Dawson, Phys. Rev. A 42, 1976 (1990).

[3] W. G. Hoover and F. H. Ree, J. Chem. Phys. 49, 3609 (1968).

[4] H.-P. Büchler, E. Demler, M. Lukin, A. Micheli, N. Prokof'ev, G. Pupillo, and P. Zoller, Phys. Rev. Lett. 98, 060404 (2007).

[5] C. Mora, O. Parcollet, and X. Waintal, Phys. Rev. B 76, 064511 (2007).

[6] W. Lechner, H.-P. Büchler, and P. Zoller, Phys. Rev. Lett. 112, 255301 (2014).

[7] G. M. Bruun and D. R. Nelson, Phys. Rev. B 89, 094112 (2014).

[8] D. Breyel, T. L. Schmidt, and A. Komnik, Phys. Rev. A 86, 023405 (2012).

[9] G. Pupillo, A. Micheli, M. Boninsegni, I. Lesanovsky, and P. Zoller, Phys. Rev. Lett. 104, 223002 (2010).

[10] J. Schachenmayer, I. Lesanovsky, A. Micheli, and A. J. Daley, New J. Phys. 12, 103044 (2010).

[11] T. Pohl, E. Demler, and M. D. Lukin, Phys. Rev. Lett. 104, 043002 (2010).

[12] R. M. W. van Bijnen, S. Smit, K. A. H. van Leeuwen, E. J. D. Vredenbregt, and S. J. J. M. F. Kokkelmans, J. Phys. B: At., Mol. Opt. Phys. 44, 184008 (2011).

[13] I. Lesanovsky, Phys. Rev. Lett. 106, 025301 (2011).

[14] N. Henkel, F. Cinti, P. Jain, G. Pupillo, and T. Pohl, Phys. Rev. Lett. 108, 265301 (2012).

[15] D. Petrosyan, Phys. Rev. A 88, 043431 (2013).

[16] W. Lechner and P. Zoller, Phys. Rev. Lett. 115, 125301 (2015).

[17] Z. Lan, J. Minář, E. Levi, W. Li, and I. Lesanovsky, Phys. Rev. Lett. 115, 203001 (2015).

[18] H. Weimer and H. P. Büchler, Phys. Rev. Lett. 105, 230403 (2010).
[19] E. Sela, M. Punk, and M. Garst, Phys. Rev. B 84, 085434 (2011).

[20] A. Lauer, D. Muth, and M. Fleischhauer, New J. Phys. 14, 095009 (2012).

[21] P. Schauß, J. Zeiher, T. Fukuhara, S. Hild, M. Cheneau, T. Macrì, T. Pohl, I. Bloch, and C. Gross, Science 347, 1455 (2015)

[22] D. Maxwell, D. J. Szwer, D. Paredes-Barato, H. Busche, J. D. Pritchard, A. Gauguet, K. J. Weatherill, M. P. A. Jones, and C. S. Adams, Phys. Rev. Lett. 110, 103001 (2013).

[23] G. Günter, H. Schempp, M. Robert-de-Saint-Vincent, V. Gavryusev, S. Helmrich, C. S. Hofmann, S. Whitlock, and M. Weidemüller, Science 342, 954 (2013).

[24] R. C. Teixeira, C. Hermann-Avigliano, T. L. Nguyen, T. Cantat-Moltrecht, J. M. Raimond, S. Haroche, S. Gleyzes, and M. Brune, Phys. Rev. Lett. 115, 013001 (2015).

[25] S. Baur, D. Tiarks, G. Rempe, and S. Dürr, Phys. Rev. Lett. 112, 073901 (2014).

[26] S. Bettelli, D. Maxwell, T. Fernholz, C. S. Adams, I. Lesanovsky, and C. Ates, Phys. Rev. A 88, 043436 (2013).

[27] D. Tiarks, S. Baur, K. Schneider, S. Dürr, and G. Rempe, Phys. Rev. Lett. 113, 053602 (2014).

[28] H. Gorniaczyk, C. Tresp, J. Schmidt, H. Fedder, and S. Hofferberth, Phys. Rev. Lett. 113, 053601 (2014).

[29] D. P. Fahey, T. J. Carroll, and M. W. Noel, Phys. Rev. A 91, 062702 (2015).

[30] D. Barredo, H. Labuhn, S. Ravets, T. Lahaye, A. Browaeys, and C. S. Adams, Phys. Rev. Lett. 114, 113002 (2015).

[31] S. D. Huber and H. P. Büchler, Phys. Rev. Lett. 108, 193006 (2012).

[32] B. Zhao, A. W. Glaetzle, G. Pupillo, and P. Zoller, Phys. Rev. Lett. 108, 193007 (2012). 
[33] W. Li, D. Viscor, S. Hofferberth, and I. Lesanovsky, Phys. Rev. Lett. 112, 243601 (2014).

[34] P. Bak and R. Bruinsma, Phys. Rev. Lett. 49, 249 (1982).

[35] E. Levi, J. Minář, J. P. Garrahan, and I. Lesanovsky, New J. Phys. 17, 123017 (2015).

[36] E. Levi, J. Minář, and I. Lesanovsky, J. Stat. Mech. Theor. Exp. (2016) 033111.

[37] See Supplemental Material at http://link.aps.org/supplemental/ 10.1103/PhysRevA.94.051603 for an introduction to the method of irreducible blocks.

[38] T. Morita, J. Phys. A 7, 289 (1974).

[39] B. Vermersch, M. Punk, A. W. Glaetzle, C. Gross, and P. Zoller, New J. Phys. 17, 013008 (2015).

[40] A. De Pasquale and P. Facchi, Phys. Rev. A 80, 032102 (2009).

[41] A. Kay and D. G. Angelakis, Europhys. Lett. 84, 20001 (2008).

[42] P. Hauke, F. M. Cucchietti, A. Müller-Hermes, M.-C. Bañuls, J. I. Cirac, and M. Lewenstein, New J. Phys. 12, 113037 (2010).

[43] A. V. Gorshkov, S. R. Manmana, G. Chen, J. Ye, E. Demler, M. D. Lukin, and A. M. Rey, Phys. Rev. Lett. 107, 115301 (2011).

[44] F. Pinheiro, G. M. Bruun, J.-P. Martikainen, and J. Larson, Phys. Rev. Lett. 111, 205302 (2013).

[45] A. W. Glaetzle, M. Dalmonte, R. Nath, C. Gross, I. Bloch, and P. Zoller, Phys. Rev. Lett. 114, 173002 (2015).

[46] R. M. W. van Bijnen and T. Pohl, Phys. Rev. Lett. 114, 243002 (2015).

[47] P. Schauß, M. Cheneau, M. Endres, T. Fukuhara, S. Hild, A. Omran, T. Pohl, C. Gross, S. Kuhr, and I. Bloch, Nature (London) 491, 87 (2012).
[48] T. M. Weber, M. Höning, T. Niederpr"m, T. Manthey, O. Thomas, V. Guarrera, M. Fleischhauer, G. Barontini, and H. Ott, Nat. Phys. 11, 157 (2015).

[49] H. Labuhn, S. Ravets, D. Barredo, L. Béguin, F. Nogrette, T. Lahaye, and A. Browaeys, Phys. Rev. A 90, 023415 (2014).

[50] K. M. Maller, M. T. Lichtman, T. Xia, Y. Sun, M. J. Piotrowicz, A. W. Carr, L. Isenhower, and M. Saffman, Phys. Rev. A 92, 022336 (2015).

[51] M. Viteau, M. G. Bason, J. Radogostowicz, N. Malossi, D. Ciampini, O. Morsch, and E. Arimondo, Phys. Rev. Lett. 107, 060402 (2011).

[52] S. E. Anderson, K. C. Younge, and G. Raithel, Phys. Rev. Lett. 107, 263001 (2011).

[53] L. Béguin, A. Vernier, R. Chicireanu, T. Lahaye, and A. Browaeys, Phys. Rev. Lett. 110, 263201 (2013).

[54] L. Li, Y. O. Dudin, and A. Kuzmich, Nature (London) 498, 466 (2013).

[55] E. Urban, T. A. Johnson, T. Henage, L. Isenhower, D. D. Yavuz, T. G. Walker, and M. Saffman, Nat. Phys. 5, 110 (2009).

[56] A. Gaëtan, Y. Miroshnychenko, T. Wilk, A. Chotia, M. Viteau, D. Comparat, P. Pillet, A. Browaeys, and P. Grangier, Nat. Phys. 5, 115 (2009).

[57] H. Gorniaczyk, C. Tresp, P. Bienias, A. Paris-Mandoki, W. Li, I. Mirgorodskiy, H. P. Büchler, I. Lesanovsky, and S. Hofferberth, Nat. Commun. 7, 12480 (2016).

[58] M. O. Scully, E. S. Fry, C. H. Raymond Ooi, and K. Wódkiewicz, Phys. Rev. Lett. 96, 010501 (2006).

[59] B. Olmos and I. Lesanovsky, Phys. Rev. A 82, 063404 (2010).

[60] Y. O. Dudin and A. Kuzmich, Science 336, 887 (2012). 